\title{
Heat and mass transfer process modeling in the packing columns and tubular absorbers
}

\author{
Mikhail Shilyaev ${ }^{1, *}$, Elena Khromova $^{1}$, and Aleksandr Bogomolov ${ }^{2,3}$ \\ ${ }^{1}$ Tomsk State University of Architecture and Construction, 634003, Tomsk, Russia \\ ${ }^{2}$ Kutateladze Institute of Thermophysics SB RAS, 630090, Novosibirsk, Russia \\ ${ }^{3}$ Gorbachev Kuzbass State Technical University, 650000, Kemerovo, Russia
}

\begin{abstract}
The mathematical models of heat and mass transfer in the packing columns (PC) and tubular absorbers (TA) with the square and triangular tube arrangement along their axes are derived for fully open flow cross-section of the device and closed tube space. The paper shows the energy advantage of TA in comparison with PC with the same extraction efficiencies of target gas component from the stream.
\end{abstract}

\section{Introduction}

The known empirical generalized volumetric mass transfer coefficients for the packing columns (PC) and tubular absorbers (TA) cannot adequately reflect the actual process for the following reasons. All of the above suggests that optimizing the regime and geometrical parameters of the PC and the TA based on the experimental factors $\beta_{i v}$, is impossible, like it is impossible to compare different vehicles (in terms of their performance, power, and size) with each other. The development of mathematical models for PC and TA will make it possible to include these devices in the general system of calculation and selection of optimal operating conditions.

\section{Problem statement}

A granular layer of thickness $l$ and porosity $\varepsilon$, consisting of the balls with diameter $d_{\mathrm{a}}$, irrigated by liquid with irrigation coefficient $q_{1}, \mathrm{~m}^{3} / \mathrm{m}^{3}$, and initial temperature $T_{10}$ is considered for the packing columns (PC). A vapor-gas flow is fed into the layer together with liquid with velocity $u_{0}$ and temperature $T_{00}$, which interacts simultaneously with the liquid film on the surfaces of layer granules in the pore channels during the heat and mass transfer processes; so, the gas components are completely absorbed from the vapor-gas flow. Under the certain thermodynamic conditions occurring locally in the pore channels, the film can evaporate or it can condense liquid vapor. The stationary, one-dimensional equations of conservation are written for the packing, taking into account the liquid film thickness on its elements, and for the vapor-gas flow along the pore channels.

The equation of heat transfer between the layer and liquid is:

* Corresponding author: shmi@mail.tomsknet.ru 


$$
\begin{gathered}
\frac{d}{d x}\left[c_{1} \rho_{1} \varepsilon_{1}\left(T_{1}-T_{0}\right)+c_{\mathrm{m}} \rho_{\mathrm{m}}(1-\varepsilon)\left(T_{\mathrm{m}}-T_{0}\right)\right]=q+\Sigma r_{i} g_{i}, \\
T_{1}=T_{10}, T_{\mathrm{m}}=T_{\mathrm{m} 0} \quad \text { при } \tau=0, \quad 0 \leq x \leq l,
\end{gathered}
$$

where $c_{1}$ and $c_{\mathrm{m}}, \rho_{1}$ and $\rho_{\mathrm{m}}, T_{1}$ and $T_{\mathrm{m}}$ are thermal capacities, densities, mean-mass temperatures of liquid and ball packing elements; $T_{0}=273 \mathrm{~K} ; r_{\mathrm{i}}$ are specific heats of evaporation-condensation of liquid and absorption-desorption of the target gas components, $\mathrm{J} / \mathrm{kg} ; q, \mathrm{~W} /\left(\mathrm{m}^{3} \mathrm{~s}\right)$, and $g_{\mathrm{j}}, \mathrm{kg} /\left(\mathrm{m}^{3} \mathrm{~s}\right)$ are heat and mass fluxes between the liquid film, balls and filtration vapor-gas flow in the packing pores:

$$
\begin{gathered}
q=-\alpha a\left[T_{\mathrm{w}}-T\right], \\
g_{i}=-\beta_{i} a\left[\rho_{\mathrm{w} i}-\rho_{i}\right],
\end{gathered}
$$

where $\alpha$ is the heat transfer coefficient, $\mathrm{W} /\left(\mathrm{m}^{2} \mathrm{~K}\right) ; a$ is the specific packing surface, $1 / \mathrm{m}$ :

$$
a=\frac{6}{d_{\mathrm{e}}}\left(1-\varepsilon^{\prime}\right), \varepsilon^{\prime}=\varepsilon-\varepsilon_{1},
$$

$d_{\mathrm{e}}$ is the equivalent diameter of the packing elements, for balls $d_{\mathrm{e}}=d_{\mathrm{a}} ; T_{\mathrm{w}}$ is the temperature on the film surface, $K$; it takes into the account of the smallness of the liquid film thickness $T_{1}$, Moreover, the temperature of the ball surface $T_{\mathrm{w}}$ it taken equal to temperature $T_{\mathrm{m}} ; T$ is the temperature of the vapor-gas flow in pores, $K ; \beta_{\mathrm{i}}$ is the mass transfer coefficient, $\mathrm{m} / \mathrm{s}$; $\rho_{\mathrm{wi}}$ and $\rho_{\mathrm{i}}$ are partial densities of the vapor-gas components near the film surface at the saturation temperature and in the filtration flow in pores, $\mathrm{kg} / \mathrm{m}^{3}$, calculated from the state equations; $\varepsilon_{1}$ is the volumetric concentration of liquid in the pore channels.

The equation of heat transfer between the filtration flow and the packing is:

$$
\rho \frac{d\left(c\left(T-T_{0}\right)\right)}{d \tau}=-q, \quad T=T_{00} \quad \text { at } \tau=0, \quad 0 \leq x \leq l
$$

where $c$ is heat capacity of the vapor-gas flow:

$$
c=\frac{\sum \rho_{\mathrm{i}} c_{\mathrm{i}}}{\rho}, \rho=\sum \rho_{\mathrm{i}} .
$$

The equation for liquid concentration is:

$$
\frac{d \varepsilon_{1}}{d \tau}=\frac{1}{\rho_{1}} \sum \beta_{\mathrm{i}} a\left(\rho_{\mathrm{wi}}-\rho_{\mathrm{i}}\right), \quad \varepsilon_{1}=\varepsilon_{10} \quad \text { at } \tau=0, \quad 0 \leq x \leq l
$$

At that, the liquid film thickness is

$$
\delta=\frac{1}{6} \frac{\varepsilon_{1}}{(1-\varepsilon)} d_{\mathrm{a}}
$$

The film velocity and its thickness were calculated using the solution of the hydrodynamic problem of gravitational film flow, when it is subjected to an external filtration flow at direct and counter flow, and continuity equation for liquid, considering evaporation-condensation processes. The local heat transfer coefficients were determined by the limit criteria theoretical dependence of M.A. Goldshtik, the local mass transfer coefficients were determined using the experimental criteria dependencies of M.E. Aerov, O. M. Todes, D. A. Narinskiy $\left(\beta_{\mathrm{i}}\right)$, and the known theoretical dependence for film mass transfer $\left(\beta_{\mathrm{iT}}\right)$. The conditions of phase equilibrium were set at the interface (the film surface - the vapor-gas flow) according to the Henry's law, and for water vapor, they were set using the well-known approximation of experimental data on the partial pressures at the state of saturation for the film temperature. The temperature of ball surface and film temperature were taken equal; at that, the first was calculated from the approximate balance equation by the heat fluxes on ball surfaces without solving the conjugate problem. The heat effect of 
water evaporation-condensation was taken equal to $r=2500 \mathrm{~kJ} / \mathrm{kg}$, for the process of gas absorption, it was taken according to V.M. Ramm. The pressure drop on the packing layer $\Delta P$ was calculated by the Ergan formula considering film thickness $\delta$ on the ball surface and energy spent for initiation of the liquid film motion. The recovery ratio of the $i^{\text {th }}$ gas component from the vapor-gas flow to liquid film $\eta_{i}$ was calculated from the ratio of mass flow rates of this component at the inlet and outlet of the pore channel:

$$
\eta_{\mathrm{i}}=1-\frac{\left[\left(\rho_{\mathrm{i}} u_{\mathrm{f}}\right)\left(\varepsilon-\varepsilon_{1}\right)\right]_{1}}{\left[\left(\rho_{\mathrm{i}} u_{\mathrm{f}}\right)\left(\varepsilon-\varepsilon_{1}\right)\right]_{0}} .
$$

Here $u_{\mathrm{f}}$ is the velocity of the vapor-gas flow in the pore channels:

$$
u_{\mathrm{f}}=u_{\mathrm{f} 0} \frac{\rho_{\mathrm{g} 0}}{\rho_{\mathrm{g}}} \frac{\varepsilon-\varepsilon_{10}}{\varepsilon-\varepsilon_{1}}, \quad u_{\mathrm{f} 0}=\frac{u_{0}}{\varepsilon-\varepsilon_{10}},
$$

$\rho_{\mathrm{g}}$ and $\rho_{\mathrm{i}}$ were calculated from continuity equation

$$
\frac{\partial \rho_{\mathrm{i}}}{\partial \tau}+\operatorname{div}\left(\rho_{\mathrm{i}} \vec{u}_{\mathrm{f}}\right)=-g_{\mathrm{i}}, \rho_{\mathrm{i}}=\rho_{\mathrm{i} 0} \quad \text { at } \tau=0, \quad 0 \leq x \leq l
$$

For $\rho_{\mathrm{g}}$, the right part of equation (10) is zero.

Similarly, as for the PC, for a tubular absorber (TA) the equations of conservation are written for the tubular absorber (TA) in the same manner as for the PC. At that, the mathematical model of the process of gas absorption in a TA is considered for two patterns of tube arrangement: square cross-section and maximally compacted, triangular, according to the centers of inner diameters of tubes $d_{\mathrm{aT}}$ (Fig. 1).
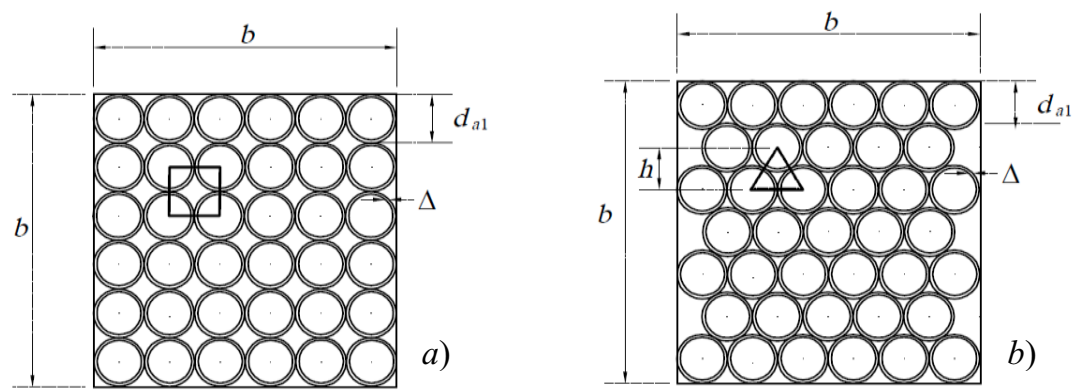

Fig. 1. Two patterns of tube arrangement in the cross-section of the working volume of TA: $a$ ) square location of tube axes; $b$ ) triangular location of tube axes.

\section{Results and discussion}

Results of calculations of mass concentration of $\mathrm{SO}_{2}$ in the water film on the grains along the pore channel length are shown in Fig. 2, a.

According to this figure, in this case, the process reaches the state of thermodynamic equilibrium at a distance from the inlet of $0.2 \mathrm{~m}$, and it is unreasonable to obtain a thicker layer. It is also clear that in the state of thermodynamic equilibrium, calculations of the film and locally volumetric mass transfer mechanisms give the same result at the same distance from the inlet. 

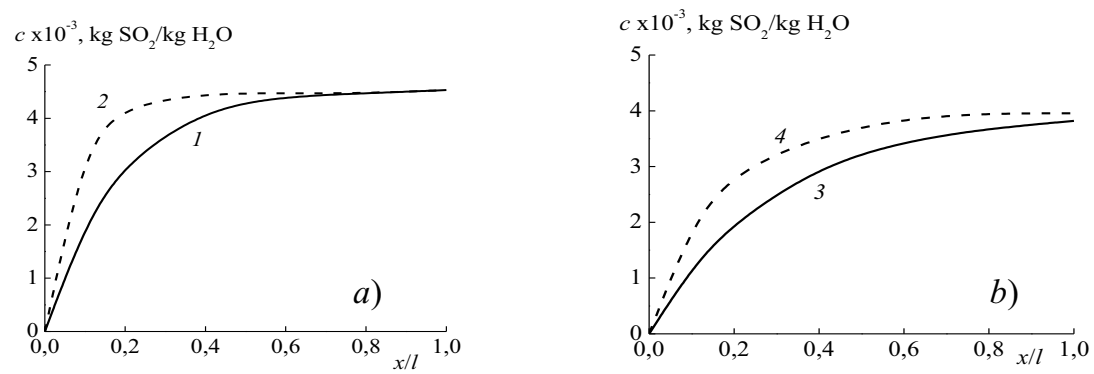

Fig. 2. Calculation results on $\mathrm{SO}_{2}$ absorption on water in a $\mathrm{PC}$ at the direct flow $(a)$ and comparison of parameters of $\mathrm{SO}_{2}$ absorption in TA with a closed intertube space and triangular tube arrangement and in a PC for corresponding conditions $(b): l=0.2 \mathrm{~m}, T_{00}=293 \mathrm{~K}, T_{10}=288 \mathrm{~K}, u_{0}=2 \mathrm{~m} / \mathrm{s}, d_{\mathrm{wv}, 0}=0.01$ $\mathrm{kg} / \mathrm{kg}$ of dry air (for water vapor), $d_{\mathrm{SO}_{2}, 0}=0.1 \mathrm{~kg} / \mathrm{kg}$ of dry air, $q_{1}=0.00953 \mathrm{~m}^{3} / \mathrm{m}^{3} ; 1-$

$\beta_{\mathrm{iэ}}=\mathrm{Nu}_{\mathrm{ei}}\left(D_{\mathrm{i}} / d_{\mathrm{ep}}\right), 2-\beta_{\mathrm{iT}}=3.41\left(D_{\mathrm{i}} / \delta\right), d_{\mathrm{a}}=0.02 \mathrm{~m}, \Delta \mathrm{P}=978 \mathrm{~Pa}, \eta_{\mathrm{SO}_{2}}=0.13986, \delta=158 \mu \mathrm{m} ; 3-$ Pc (used $\left.\left(\beta_{\mathrm{ig}}\right)\right): d_{\mathrm{ae}}=0.0097836 \mathrm{~m}, \varepsilon=0.734 \mathrm{~m}^{3} / \mathrm{m}^{3}, \eta_{\mathrm{SO}_{2}}=0.2257, \Delta P_{1}=138 \mathrm{~Pa} ; 4-\mathrm{TA}$ (used $\left(\beta_{\mathrm{iT}}\right)$ ) $d_{\mathrm{aT}}=0.02 \mathrm{~m}, \eta_{\mathrm{SO}_{2}}=0.22466, \Delta \mathrm{P}_{2}=1.94 \mathrm{~Pa}$.

Calculations show that from the energy consumption viewpoint for gas cleaning, the direct and counter flow schemes with the same efficiencies of gas component extraction are equal. However, the preference should be given to the direct-flow scheme, which excludes flooding regime and allows absorption in the $\mathrm{PC}$ at higher velocities of the cleaned gas, i.e. at significantly higher flow rates through a single apparatus.

Calculation results of the absorption process in TA are shown in Fig. 2, $b$ in comparison with ND under the appropriate conditions. At that, the transition is performed by the formula for the spherical elements of packing with the equivalent diameter:

$$
d_{\mathrm{ae}}=\frac{6}{a}(1-\varepsilon) \text {. }
$$

As seen from the figure, calculation results for PC and TA are in satisfactory agreement with each other in terms of the efficiency of $\mathrm{SO}_{2}$ extraction on water. However, in terms of the pressure drop, the advantage of TA over PC is obvious. It is shown that it is extremely difficult, almost impossible to distribute evenly the liquid in the form of a film over the inner and outer tube surfaces, when the efficiency of pattern with the outer intertube space prevails over the pattern with the closed intertube space. The second pattern is more preferable. The stable and uniform distribution of the film over the inner surfaces of tubes can be easily arranged by rotating the liquid, sprayed at the inlet, with a screw device inside the tubes, which creates rotational movement of the vapor-gas flow. Due to the action of the arising centrifugal forces, the dispersed liquid droplets are thrown to the walls of tubes, forming there a rotating film.

In addition, the intertube space could be used for tubes cooling to maintain their temperatures at the lowest level contributing to more efficient extraction of the target gas component on the absorbent.

\section{Conclusion}

The proposed models can be used in engineering calculations of heat and mass transfer of PC, TA and gas-cleaning devices. 